\title{
Análisis de la casuística equina del área de Grandes Animales de la Clínica Veterinaria U.D.C.A, Bogotá, Colombia
}

\section{Analysis of equine casuistic in the Large Animals area of the Veterinary Clinic U.D.C.A, Bogotá, Colombia}

\author{
Sandra Stella Ujueta Rodríguez
}

'MVZ. M.Sc. Microbiología y Epidemiología. Universidad de Ciencias Aplicadas y Ambientales U.D.C.A, Calle 222 No. 57-30, Bogotá, D.C., Colombia, e-mail: sujueta@udca.edu.co, (Dhttps://orcid.org/0000-0002-1134-9924

Cómo citar: Ujueta Rodríguez, S.S. 2019. Análisis de la casuística equina del área de Grandes Animales de la Clínica Veterinaria U.D.C.A, Bogotá, Colombia. Rev. U.D.C.A Act. \& Div. Cient. 22(1):e1219. https://doi.org/10.31910/rudca.v22.n1.2019.1219

Artículo de acceso abierto publicado por Revista U.D.C.A Actualidad \& Divulgación Científica bajo una licencia Creative Commons CC BY-NC 4.0

Recibido: Diciembre 7 de 2018

Aceptado: Mayo 13 de 2019

\section{RESUMEN}

Conocer las patologías más frecuentes facilita la orientación clínica mejorando el pronóstico de los pacientes. Para determinar las patologías que se presentan en caballos (Equus ferus caballus) en la Clínica Veterinaria U.D.C.A, se realizó un estudio retrospectivo, entre enero y diciembre de 2017. Se analizaron 242 historias, clasificando los casos, según la fecha, procedencia, raza, sexo, edad, diagnóstico definitivo, sistema, tratamiento médico-quirúrgico y evolución. La procedencia más frecuente fue del departamento de Cundinamarca y de Bogotá; 55,8\% fueron hembras, 44,2\% machos, con edad entre 1 día y 25 años; $24 \%$ jóvenes, $76 \%$ adultos, con edad promedio de 81,5 meses y desviación estándar de 51,7 meses; la raza predominante fue Caballo Criollo Colombiano (CCC). Los sistemas más afectados fueron músculo esquelético (SME), digestivo (SD), y reproductivo; los casos más frecuentes fueron Arpeo idiopático o hipertonía equina refleja, que ocurrió únicamente en CCC; alteraciones del intestino grueso, y criptorquidia, respectivamente. Tratamiento quirúrgico, se dio a $66,5 \%$ de los casos, principalmente, a los del SME, digestivo y reproductivo. La mortalidad fue de 13,7\%; fallecieron 13 equinos y a 22, se les practicó eutanasia; los casos mortales, se presentaron principalmente en los del SD, relacionados con cólico y ninguno en los de los sistemas respiratorio, órganos de los sentidos y tegumentario. La casuística es amplia y variada; el SME fue el más afectado en los caballos. El compromiso funcional y vital para los animales hace necesario investigar sobre factores de riesgo del Arpeo idiopático y cólico equino.

Palabras clave: caballos; casos clínicos; arpeo idiopático; cólico; criptorquidia.

\section{ABSTRACT}

Know the most frequent pathologies facilitates the clinical orientation improving the prognosis of the patients. To determine the pathologies that occur in horses at the U.D.C.A Veterinary Clinic, a retrospective study was conducted between january and december 2017. 242 clinical histories were analyzed, classifying the cases according to date, origin, race, sex, age, definitive diagnosis, system, medical-surgical treatment and evolution. The most frequent source was the department of Cundinamarca and the city of Bogotá, $55.8 \%$ were females, $44.2 \%$ males, age between 1 day and 25 years, $24 \%$ young, $76 \%$ adults with an average age of 81.5 months and a standard deviation of 51.7 months, the predominant breed was Colombian Creole Horse (CCC). The most affected systems were muscle skeletal (ME), digestive (D), and reproductive, the most frequent cases were classic stringhalt or equine reflex hypertonia, which occurred only in CCC, alterations of the large intestine, and cryptorchidism, respectively. Surgical treatment was given to $66.5 \%$ 
Ujueta Rodríguez, S.S.: Casuística en equinos

of the cases, mainly to SME, digestive and reproductive cases. Mortality was $13.7 \%, 13$ equines died and 22 were euthanized, fatal cases occurred mainly in those of SD related to colic and none in the respiratory, organs of the senses and integumentary systems. The casuistic is wide and varied, the MES was the most affected in the horses attended, the functional and vital commitment for the animals makes it necessary to investigate the risk factors of the classic stringhalt and equine colic.

Keywords: equines; clinical cases; classic stringhalt; colic; criptorquidism.

\section{INTRODUCCIÓN}

Aunque son pocos los estudios reportados sobre casuística clínica en equinos en Colombia, la casuística en clínicas veterinarias de la Universidad CES y la de la Corporación Universitaria Lasallista, ubicadas en el departamento de Antioquia, Colombia, ha mostrado que los sistemas más afectados en equinos son el músculo esquelético, derivando en manejo quirúrgico, seguido por el digestivo (Henao et al. 2010; Jaramillo et al. 2017). En tanto, que los sistemas digestivo y reproductivo fueron los más afectados, en una clínica veterinaria particular, de la Estrella, Antioquia (Ortiz et al. 2013) y el sistema de órganos de los sentidos, el menos alterado, en los tres centros especializados.

En Colombia, las afecciones más frecuentes en equinos de vaquería, según los servicios de clínica ambulatoria de la Universidad de Córdoba, han sido las alteraciones dermatológicas, los problemas digestivos, los procedimientos quirúrgicos, las alteraciones locomotoras (Cardona et al. 2017) y, el sistema hematopoyético, seguido por el tegumentario, digestivo y los procedimientos quirúrgicos, en general (Buitrago et al. 2017). En las afecciones del sistema digestivo en el caballo criollo de trabajo, del departamento de Caldas, son prevalentes las alteraciones dentarias, según el servicio ambulatorio de la Universidad de Caldas (Cruz et al. 2012). Entretanto, la casuística en equinos del área quirúrgica de este centro ha perdido importancia respecto a las especies canina y felina, por su ubicación y por la transformación de zona rural a urbana, del área de influencia de la clínica (Franco \& Silva-Molano, 2009).

Los pacientes atendidos en centros especializados y en los servicios ambulatorios del país muestran predominio del caballo Criollo Colombiano y las hembras, con variación en la edad y tendencia a la edad adulta (Henao et al. 2010; Cruz et al. 2012; Ortiz et al. 2013; Jaramillo et al. 2017). La mortalidad reportada solo para los animales tratados quirúrgicamente es variable, entre 85,1 y 9,2\% (Ortiz et al. 2013; Jaramillo et al. 2017).

El área de grandes animales de la Clínica Veterinaria de la Facultad de Ciencias Agropecuarias de la Universidad de Ciencias Aplicadas y Ambientales U.D.C.A, Bogotá, que ofrece servicios desde 1988, carece de información sobre la casuística, que permita mejorar el pronóstico de los pacientes, en el marco de la formación académica y del ofrecimiento del servicio a sus usuarios. El objetivo del presente estudio fue identificar las patologías más frecuentes, tipo de tratamiento y evolución de los casos clínicos en caballos, atendidos en la clínica, en 2017, para analizar, constructivamente, los casos más sobresalientes, establecer comparativos con otros centros, mejorar la planificación y optimizar el servicio e, incluso, identificar prioridades de investigación sobre patologías de interés en equinos.

\section{MATERIALES Y MÉTODOS}

Se realizó un estudio descriptivo retrospectivo de la casuística de equinos atendidos por el área de grandes animales de la Clínica Veterinaria de la Facultad de Ciencias Agropecuarias de la Universidad de Ciencias Aplicadas y Ambientales U.D.C.A, Bogotá, Colombia, entre el primero de enero y el 31 de diciembre de 2017. Los casos atendidos, se clasificaron según la fecha de consulta, procedencia, especie, raza, sexo, edad, diagnóstico definitivo, sistema afectado, tipo de tratamiento (médico-quirúrgico) y evolución.

Los datos fueron registrados y procesados en una base de datos elaborada en el programa Microsoft Office Excel $2013^{\circledR}$ y analizados en forma descriptiva, determinando frecuencias, promedios y desviación estándar, para las variables propuestas. Para el análisis estadístico, se codificaron binariamente las enfermedades por sistema con cero $(0)=$ ausencia, o uno $(1)=$ presencia, el sexo con cero $(0)=$ macho o uno $(1)=$ hembra. La relación entre la presencia de enfermedad por sistema, el sexo y la edad, se analizó mediante ji-cuadrado $\left(\mathrm{X}^{2}\right)$, usando el programa Epi-Info versión 6 de dominio público (OPS).

La procedencia y la edad de los pacientes fue suministrada por los propietarios de los animales y los diagnósticos definitivos correspondieron con los registrados por el médico tratante. Se garantizó la confidencialidad sobre el nombre de los pacientes y sus propietarios.

Según la resolución 8430 de 1993 Ministerio de Salud de Colombia, el estudio es considerado sin riesgo para los animales, porque es documental retrospectivo. El estudio cuenta con el aval de la Universidad y la Facultad de Ciencias Agropecuarias, que suministró los documentos.

\section{RESULTADOS Y DISCUSIÓN}

De 247 historias clínicas analizadas, 242 cumplieron con los criterios de inclusión en el estudio, diligenciamiento claro y completo. Durante el periodo, mensualmente, se atendieron entre 14 y 42 casos, con promedio de 20,2 y desviación estándar de 7,7; 121 casos fueron urgencias, con promedio de 10,1 y desviación estándar de 4,9, urgencias por mes. Los pacientes, en su mayoría, fueron remitidos por profesionales, que consideraron la atención especializada, como una alternativa necesaria.

Sexo, edad, raza y procedencia: De los 242 caballos, 135 fueron hembras y 107 machos; la edad fluctuó entre 1 día y 25 años; 184 fueron adultos, de 36 meses o más (según madurez sexual), de los cuales, 7 fueron senior ( $\geq 216$ meses, 18 años) y 58 equinos jóvenes. La edad promedio de los adultos fue de 81,5 meses (6,8 años), con 
desviación estándar de 51,7 meses (4,3 años). Estas observaciones corresponden con el grupo de equinos adultos, como el de mayor casuística en una clínica veterinaria universitaria de la ciudad de Medellín, Colombia (Henao et al. 2010).

La mayoría fueron Caballo Criollo Colombiano (CCC) (77,7\%), seguido por las razas Cuarto de Milla (6,6\%), Español o Andaluz (2,9\%), Silla Argentino (2,5\%), Shire y Percherón (2,1\% cada una), Pinto Americano y Ponies de varias razas (1,2\% cada una), Lusitana y Silla Holandés $(0,8 \%$ cada una). Las razas de las que menos llegaron casos en el periodo fueron Frisón, Belga, Appaloosa, Árabe, y Polo Argentino con 1 caso cada una (0,4\%). Coincidente con la atención de la especie equina y la raza Criollo Colombiano en centros veterinarios especializados del país, encontrada por Ortiz et al. (2013), siendo, según Gómez et al. (2007) la población de equinos de Cundinamarca representativa de la población equina colombiana, en el presente estudio, se observó el predominio de CCC, debido a su amplia distribución en el país.

La mayoría de pacientes provenían del departamento de Cundinamarca $(73,1 \%)$, principalmente, de los municipios de Tenjo, Subachoque, Tabio, Chía, Fusagasugá, Facatativá, Cota y La Calera, seguidos por Guasca, Sopó, Cajicá, Zipaquirá, Cogua, San Francisco, La Punta, Supatá, Tocaima, Madrid y Susa, y con menor números de casos de Vianí, La Mesa, Sesquilé, Silvania, Bojacá, Gachetá, Villeta y Villapinzón (Figura 1). De Bogotá, el 12\% de los casos y, el restante 14,9\%, de los departamentos de Meta, Tolima, Casanare, Huila, Bolívar, Quindío, Valle, Norte de Santander, Santander, Caquetá, Caldas, Boyacá, Guaviare y Antioquia.

La remisión de los pacientes, principalmente desde municipios del departamento de Cundinamarca y de Bogotá, se ve favorecida, por su ubicación en esta zona, la tercera con mayor población equina, $7,58 \%$, del censo equino nacional (ICA, 2017), que concentra fincas, criaderos, centros recreativos y de hipoterapia, las escuelas de caballería y de carabineros del Ejército y la Policía Nacional, respectivamente, así como tres de las cinco ligas ecuestres del país (F.E.C., 2017), con sus clubes, escuelas, centros de preparación y adiestramiento. También se debe al reconocimiento de los servicios en clínica equina, pues dispone de recursos físicos, equipos, de un cuadro médico de profesionales especialistas y de atención de urgencias.

Sistemas orgánicos afectados: El músculo esquelético (SME) resultó ser el sistema más afectado en equinos, seguido por el digestivo y el reproductivo. Esto coincide con lo encontrado en la clínica veterinaria Lasallista, Caldas, Colombia (Jaramillo et al. 2017). Le siguieron, el respiratorio y, en menor proporción, casos de los sistemas urinario, nervioso, inmune, cardiovascular, órganos de los sentidos y tegumentario, clasificados como otros, que representaron, en conjunto, el 10,3\% del total de casos, siendo el sistema tegumentario y los casos multisistémicos, los que mostraron menor proporción (Tabla 1 y 2).

El predominio de las patologías del SME frente a las de los demás sistemas, seguido por los problemas del sistema digestivo, tanto en hembras como en machos equinos, coincide con lo reportado por Jaramillo et al. (2017) y Henao et al. (2010), pero contradice lo reportado por Ortiz et al. (2013), quienes citan que los problemas del SME mostraron baja frecuencia de presentación en la atención clínica. Los casos, en ambos sexos, predominaron en adultos. Las hembras mostraron mayor afectación que los machos en todos los sistemas, a excepción del sistema reproductivo, en el que el mayor número de casos ocurrió en machos (Figura 2).

Sistema músculo esquelético (SME): Las patologías del SME, se presentaron más en hembras $(61,3 \%)$ y en equinos adultos $(73,6 \%)$, y no se observó ningún caso en animales senior (Figura 2). En general, no se halló asociación entre las variables sexo y edad y la presentación de afectación del SME, a un nivel de significación del 95\%, según ji cuadrado.

La mayor casuística correspondió al SME, sobresaliendo las enfermedades relacionadas con las extremidades, viéndose más afectadas las posteriores $(90,5 \%)$, en las que más frecuentemente se presentó Arpeo idiopático, seguido por traumas o fracturas e inflamaciones asociadas con artritis, desmitis y tendinitis; las afecciones de menor presentación en las extremidades fueron las desviaciones de los aplomos. Con menor frecuencia, se atendieron casos en los que se vio comprometido el abdomen, principalmente por hernias y la cabeza, por la presentación de masas y abscesos en los maxilares (Tabla 1).

El Arpeo idiopático, hipertonía equina refleja o stringhalt clásico, se presentó sin historia de eventos traumáticos, episodios nerviosos ni una causa específica, afectó a ambos miembros posteriores en el $98 \%$ de los casos y no mostró picos epizoóticos, lo que difiere del Arpeo adquirido (Australian stringhalt). Únicamente, se vieron afectados caballos Criollo Colombiano, siendo la incidencia mayor en las hembras (72\%) y en caballos adultos (74\%), menor en jóvenes (26\%), con 13 casos, de los cuales, 11 fueron hembras y ningún caso en animales senior, con un rango entre 1,3 y 8 años, un promedio de edad de 3,8 años y una desviación estándar de 1,8 años, lo que coincide con la aparición de la afección en caballos criollos en pleno adiestramiento o al inicio de este.

La proporción de Arpeo idiopático (30\%) reportada en equinos de trabajo en el departamento de Córdoba, Colombia (Buitrago et al. 2017) fue menor respecto a la encontrada en el presente estudio. Al Stringhalt clásico en Colombia, se le califica de origen idiopático (Duque et al. 2014), considerada, tradicionalmente, como una patología frecuente, heredada y exclusiva del CCC (Vega \& Martínez, 2018), sobre la cual, falta identificar, entre otros, el efecto de la exposición a ejercicios repetitivos (Martínez et al. 2007). Este efecto mecánico de una posible alteración morfológica en el CCC, se puede deber a características músculo esqueléticas, que se ve favorecido por las condiciones de manejo y el entrenamiento al que son sometidos estos animales, destinados, especialmente, a actividades deportivas y de exposición.

Las lesiones ortopédicas son muy comunes en los equinos de deporte (Morales et al. 2009) o exposición, debido a factores 


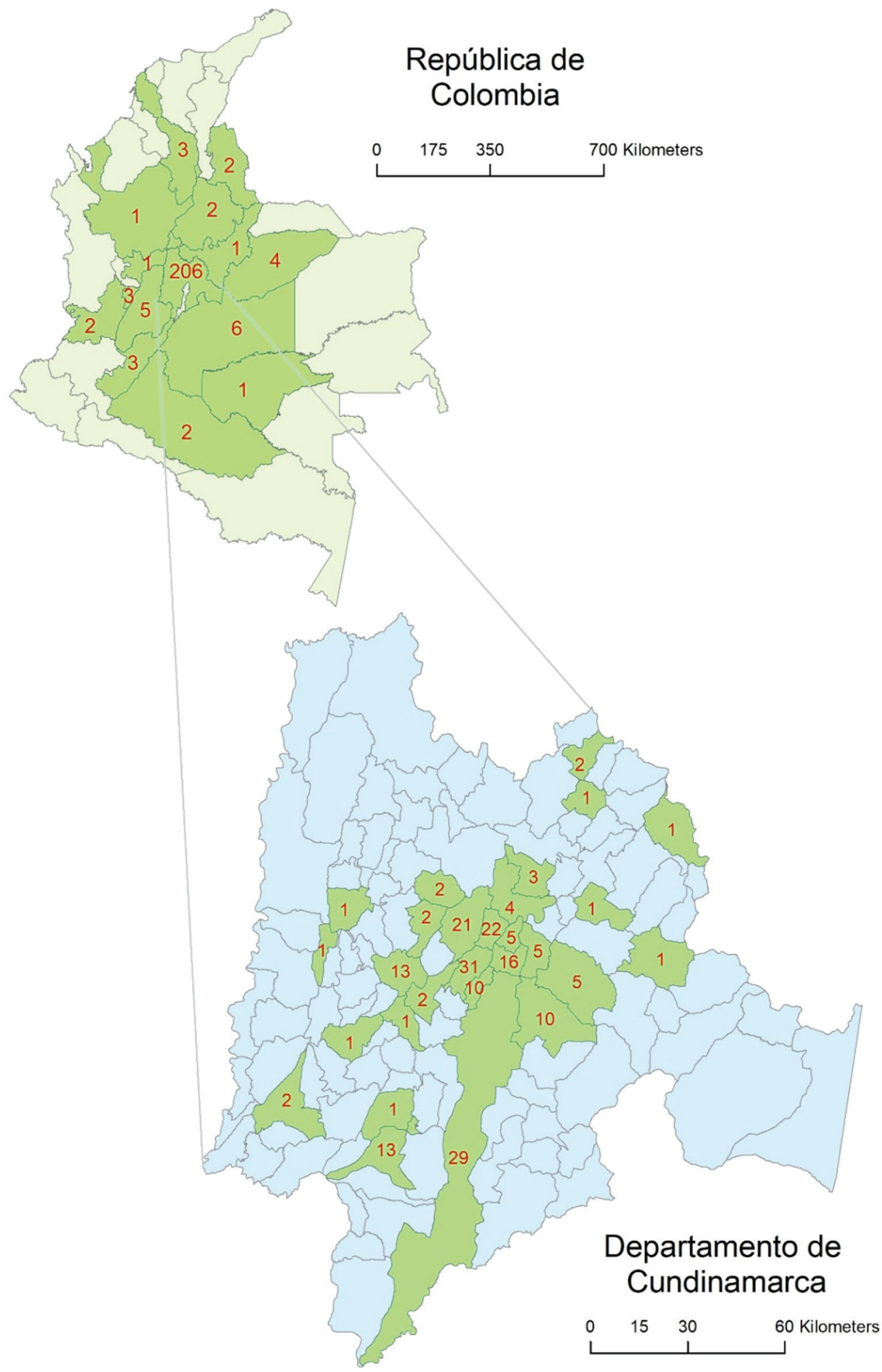

Figura 1. Casos por municipio, región y departamento. 
Tabla 1. Distribución porcentual de casos, según el diagnóstico, para los sistemas músculo esquelético y digestivo en equinos.

\begin{tabular}{|c|c|c|c|c|}
\hline \multicolumn{5}{|c|}{ Sistema músculo esquelético } \\
\hline \multirow{2}{*}{ Sección } & \multirow{2}{*}{ Diagnóstico } & \multicolumn{2}{|c|}{ casos } & \multirow{2}{*}{$\begin{array}{l}\text { casos/ } \\
\text { sección } \\
\%\end{array}$} \\
\hline & & $\mathrm{n}$ & $\%$ & \\
\hline \multirow{7}{*}{ Extremidades } & Arpeo idiopático & 50 & 47,6 & \multirow{7}{*}{90,0} \\
\hline & Traumas/fracturas & 19 & 17,9 & \\
\hline & $\begin{array}{l}\text { Procesos inflamatorios articulares, OA, desmitis y } \\
\text { tendinitis }\end{array}$ & 13 & 12,3 & \\
\hline & Masas & 5 & 4,7 & \\
\hline & Neuromas, compresiones & 3 & 2,8 & \\
\hline & Laminitis/hormiguillo, absceso subsolar & 3 & 2,8 & \\
\hline & Aplomos desviación & 2 & 1,9 & \\
\hline \multirow{2}{*}{ Abdomen } & Hernias & 7 & 6,6 & \multirow{2}{*}{8,5} \\
\hline & Trauma & 2 & 1,9 & \\
\hline Cabeza & Masa/absceso & 2 & 1,9 & 1,9 \\
\hline & Total & 106 & 100 & 100 \\
\hline \multicolumn{5}{|c|}{ Sistema digestivo } \\
\hline Boca & Absceso periodontal tercer molar & 1 & 1,4 & 1,4 \\
\hline \multirow{3}{*}{ Estómago } & Sobrecarga & 5 & 7 & \multirow{3}{*}{11,2} \\
\hline & Gastritis & 2 & 2,8 & \\
\hline & Perforación & 1 & 1,4 & \\
\hline \multirow[t]{2}{*}{ I. Delgado } & $\begin{array}{l}\text { Obstrucción por vólvulo, atrapamiento en foramen } \\
\text { epiploico, enterolito, Impactación }\end{array}$ & 7 & 9,9 & \multirow[t]{2}{*}{11,3} \\
\hline & Adherencias & 1 & 1,4 & \\
\hline \multirow{4}{*}{ I. Grueso } & Colitis & 5 & 7 & \multirow{4}{*}{67,6} \\
\hline & Necrosis & 5 & 7 & \\
\hline & $\begin{array}{l}\text { Obstrucción por vólvulo, Impactación, encarcelami- } \\
\text { ento Colon mayor, enterolito, compactación, sobre- } \\
\text { carga, intususcepción, parasitosis }\end{array}$ & 30 & 42,3 & \\
\hline & Otros: desplazamiento, distensión, aplasia, ruptura & 8 & 11,3 & \\
\hline \multicolumn{2}{|l|}{ Inespecíficas } & 6 & 8,5 & 8,5 \\
\hline & Total & 71 & 100 & 100 \\
\hline
\end{tabular}


Tabla 2. Distribución porcentual de casos, según el diagnóstico, para los sistemas reproductivo, respiratorio y otros sistemas orgánicos en equinos.

\begin{tabular}{|c|c|c|c|c|}
\hline \multicolumn{5}{|c|}{ Sistema reproductivo } \\
\hline \multirow{2}{*}{ Sección } & \multirow{2}{*}{ Diagnóstico } & \multicolumn{2}{|c|}{ Casos } & \multirow{2}{*}{ casos/ sección \% } \\
\hline & & $\mathrm{n}$ & $\%$ & \\
\hline \multirow{2}{*}{ Testículos } & Criptorquidismo & 11 & 45,8 & \multirow{2}{*}{50,1} \\
\hline & Hidrocele & 1 & 4,2 & \\
\hline \multirow{2}{*}{ Pene y prepucio } & Balanopostitis & 1 & 4,2 & \multirow{2}{*}{12,5} \\
\hline & Carcinoma escamocelular & 2 & 8,3 & \\
\hline \multirow{3}{*}{ Útero } & Torsión & 1 & 4,2 & \multirow{3}{*}{20,9} \\
\hline & Retención de placenta & 1 & 4,2 & \\
\hline & Parto distócico & 3 & 12,5 & \\
\hline Vagina & FRV & 4 & 16,7 & 16,7 \\
\hline & Total & 24 & 100 & 100 \\
\hline \multicolumn{5}{|c|}{ Sistema respiratorio } \\
\hline \multirow{5}{*}{ Vías aéreas anteriores } & Elongación del paladar blando & 4 & 25 & \multirow{5}{*}{68,8} \\
\hline & Estenosis del meato ventral & 1 & 6,3 & \\
\hline & Faringitis & 2 & 12,5 & \\
\hline & Carcinoma, infección micótica & 2 & 12,5 & \\
\hline & Absceso bolsas guturales & 2 & 12,5 & \\
\hline \multirow{2}{*}{$\begin{array}{l}\text { Vías aéreas posteri- } \\
\text { ores }\end{array}$} & Neumonía & 4 & 25 & \multirow{2}{*}{31,3} \\
\hline & ORVA & 1 & 6,3 & \\
\hline & Total & 16 & 100 & 100 \\
\hline \multicolumn{5}{|c|}{ Otros sistemas } \\
\hline Urinario & $\begin{array}{l}\text { Ruptura vesical, infección renal, Insuficiencia } \\
\text { renal, uraco persistente }\end{array}$ & 4 & 16 & 16 \\
\hline Nervioso & $\begin{array}{l}\text { Encefalitis, meningitis, cauda equina, intoxi- } \\
\text { cación }\end{array}$ & 5 & 20 & 20 \\
\hline $\begin{array}{l}\text { Órganos de los } \\
\text { Sentidos }\end{array}$ & $\begin{array}{l}\text { Herida palpebral, carcinoma palpebral, absceso } \\
\text { auricular }\end{array}$ & 4 & 16 & 16 \\
\hline Inmune & Isoeritrólisis neonatal, control de inmunización & 4 & 16 & 16 \\
\hline Cardiovascular & Hemoparásitos, linfangitis & 4 & 16 & 16 \\
\hline Tegumentario & Ectoparásitos, papilomatosis & 2 & 8 & 8 \\
\hline Multisistémico & & 2 & 8 & 8 \\
\hline & Total & 25 & 100 & 100 \\
\hline
\end{tabular}

predisponentes, como el peso, la alimentación, la edad al comienzo del adiestramiento, el aumento en el grado de dificultad durante entrenamientos y competencias, los diferentes tipos de superficies de trabajo y la conformación de los miembros (Montoya \& Botero, 2008). Algunos programas de entrenamiento y de eventos deportivos o exposiciones someten a los caballos a esfuerzos o movimientos repetitivos, que los predisponen a este tipo de lesiones (Menarim et al. 2012).
Sistema digestivo (SD): Las patologías del SD en equinos fueron más frecuentes en hembras $(56,3 \%)$ y se presentaron, principalmente, en equinos adultos $(81,7 \%)$, de los cuales, cuatro fueron senior (Figura 2), con un rango de edad entre 1 día y 24 años, un promedio de edad de siete años y una desviación estándar de 5,1 años. No se encontró asociación entre las variables sexo y edad y la presentación de afectación del $\mathrm{SD}$, a un nivel de significación, del $95 \%$, según ji cuadrado, lo que podría indicar que las alteraciones del sistema digestivo se pueden presentar en hembras y machos, de todas las edades. 


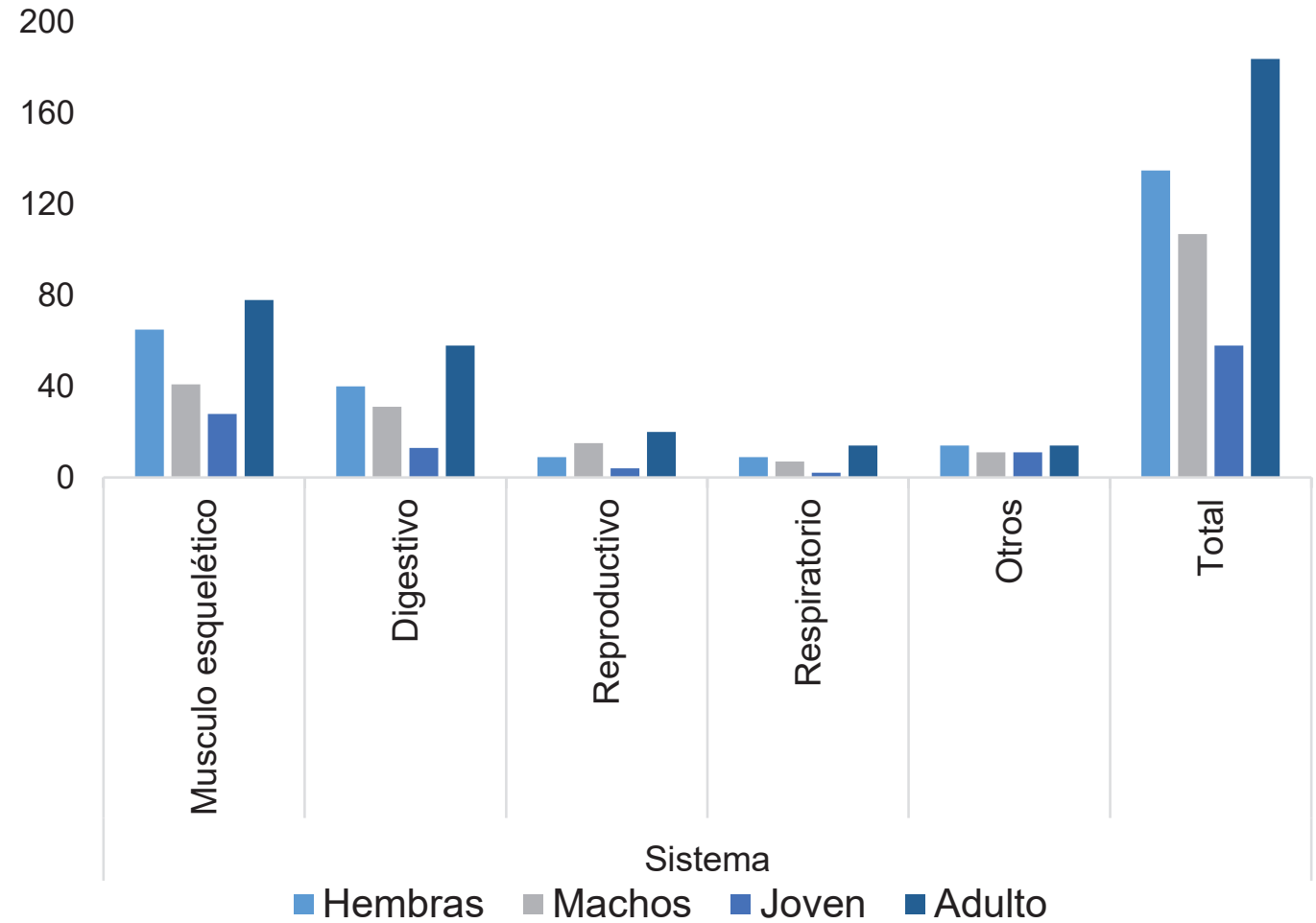

Figura 2. Número de casos por sistema según el sexo y la edad.

Los casos más sobresalientes fueron los relacionados con afecciones del intestino grueso, coincidente con lo reportado por Ortiz et al. (2013); siendo los más frecuentes los procesos obstructivos, provocados por torsiones o vólvulos, encarcelamiento nefroesplénico, enterolitos, sobrecarga, compactación, impactación, intususcepción y parasitosis (Tabla 1).

Estos resultados varían de lo reportado en estudios realizados por las Universidades de Córdoba (Buitrago et al. 2017) y Caldas (Cruz et al. 2012), que encontraron que las afecciones del sistema digestivo se deben, máxime, a alteraciones dentarias, que tienen alta prevalencia en caballos de vaquería (Cardona et al. 2018) y ocupan el tercer lugar en la casuística global de esta especie, aunque, generalmente, pasan desapercibidas (Amaya et al. 2009). La baja frecuencia de afecciones dentales, en el presente trabajo, puede estar relacionada con el manejo y los servicios médicos que reciben los animales bajo estudio respecto a los animales de trabajo.

Las afecciones del intestino delgado y estómago indicaron frecuencias similares y, menos habituales, resultaron las clasificadas como inespecíficas, porque no contaron con un diagnóstico definitivo claro, aunque presentaron el cólico abdominal, como signo clínico, que compartieron con las demás alteraciones del sistema digestivo, sin haberse visto afectados otros sistemas orgánicos (Tabla 1). Las alteraciones gástricas de menor frecuencia frente a las del intestino grueso, coinciden con lo encontrado en Antioquia, Colombia (Ortiz et al. 2013), pero difieren de lo reportado por Laranjeira et al. (2009), quienes citan como más frecuente el cólico por dilatación gástrica, en Río de Janeiro, Brasil.

Respeto a los factores predisponentes de las afecciones del SD, los estudios difieren sobre la relación entre los padecimientos de este sistema con la edad y el sexo; por ejemplo, en los casos de afecciones dentales es clara la relación directa entre la edad y la presentación de la enfermedad (Cruz et al. 2012), pero contrariamente, en alteraciones como el atrapamiento del intestino delgado, a la vez que, autores mencionan la edad avanzada, como factor predisponente, otros reportan que, además de ser poco común, no es propia de animales viejos (Duque et al. 2010; Freeman \& Schaeffer, 2001).

La diferencia entre reportes, se puede deber a la ubicación geográfica de las poblaciones de caballos estudiadas, a la línea de especialización clínica y al tipo de atención de los centros veterinarios, así como a las especialidades de los médicos veterinarios que atienden los pacientes en estos centros.

En 90,1\% (64) de los casos del sistema digestivo, el motivo de consulta fue cólico o SAA (Síndrome Abdominal Agudo), que corresponde con uno de los padecimientos más prevalentes y desafiantes para los veterinarios de equinos, pues lograr la supervivencia de pacientes con cólico es un reto, dadas las múltiples enfermedades que pueden desencadenarlo y los procesos fisiopatológicos asociados. 
Sistema reproductivo: El tercer sistema más afectado fue el reproductivo, viéndose más afectados los machos (62,5\%) adultos, entre los que se presentó un caso en un animal senior, con un rango de edad entre 2 y 27,5 años, una edad promedio de 6,7 años y una desviación estándar de 5,3 años. Como se observa en la tabla 2, la mitad de los casos estuvieron relacionados con los testículos, siendo el criptorquidismo el más frecuente, seguido por las afecciones del útero y la vagina, con casos de partos distócicos y fístula rectovaginal y, en menor proporción, se presentaron casos relacionados con el pene y prepucio. Similar situación se observó en la casuística de una clínica en Antioquia, Colombia, en la que los problemas de este sistema ocuparon el tercer lugar, siendo más frecuentes la criptorquidia, seguido por orquiectomia electiva y fístula rectovaginal (Ortiz et al. 2013).

El 9,4\% del total de los casos en machos correspondió a criptorquidismo, viéndose afectados, principalmente, adultos, lo que da idea del carácter frecuente de la alteración, relacionado con su condición heredable (Cattelan et al. 2004). La presentación de torción uterina, distocia, retención placentaria y laceraciones vaginales, correspondieron con los problemas más frecuentes en el periparto, coincidente con lo citado en la literatura (Fernandes et al. 2014).

Sistema respiratorio: La presentación de casos fue similar en machos y hembras, presentándose principalmente en adultos como se observa en la figura 2.

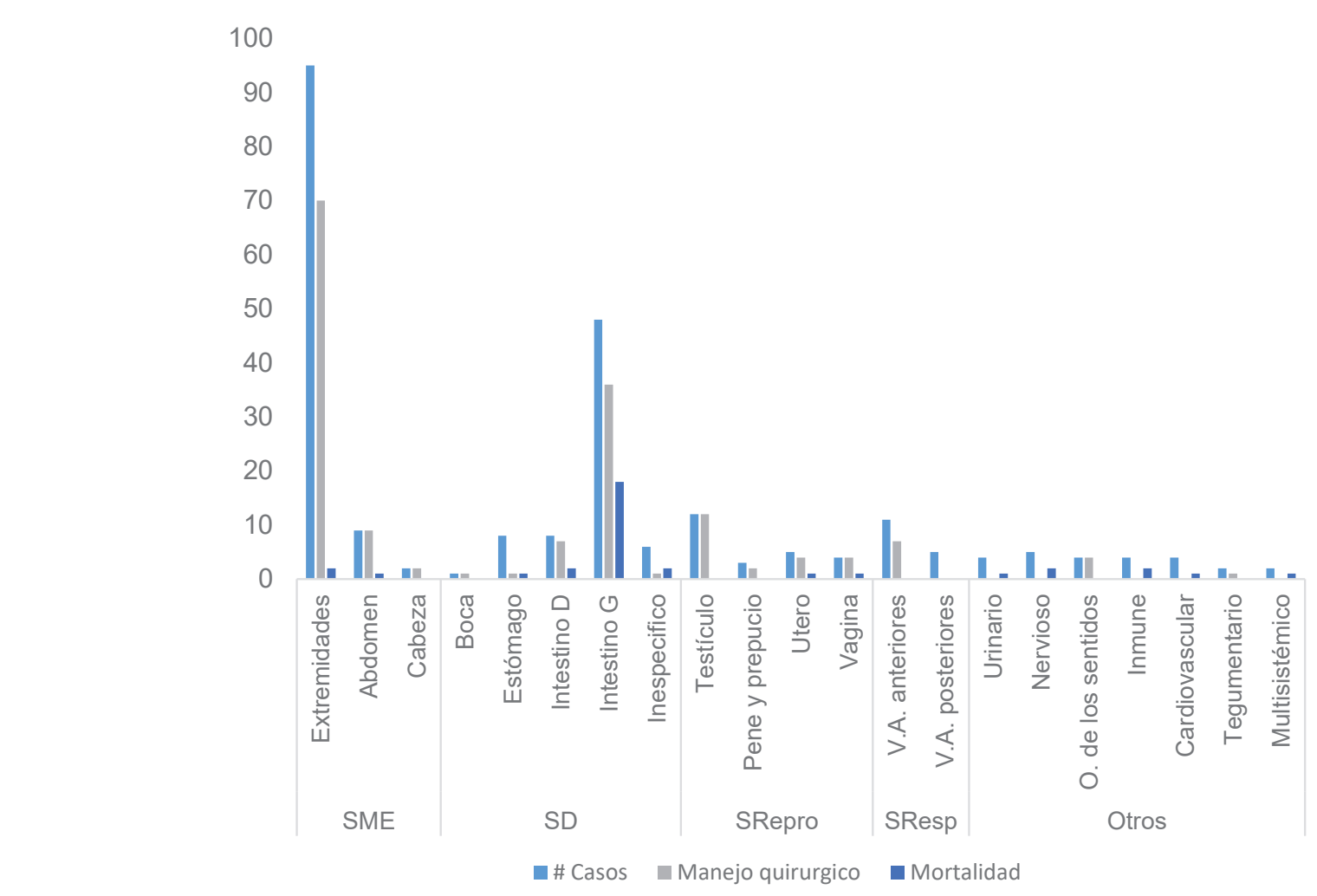

Los casos más frecuentes fueron la elongación del paladar blando y neumonía y, los de menor presentación, estenosis del meato ventral y obstrucción recurrente de las vías aéreas (ORVA), observándose más alteraciones de las vías anteriores (Tabla 2), lo que coincide con el hallazgo de frecuentes alteraciones en esta porción, según Arias et al. (2013) y Ortiz et al. (2013), aunque la menor presentación de casos de ORVA es contraria al reporte del Sistema de Vigilancia Epidemiológia del ICA (Instituto Colombiano Agropecuario), que cita a ésta, como una de las afecciones respiratorias más frecuente en la población caballar de Colombia (Castillo et al. 2013). Los casos de elongación de paladar blando, se presentaron únicamente en equinos de la raza Criollo Colombiano, similar a lo observado por Martínez \& Oliver (2012).

Entre los casos en los que se vieron afectados los demás sistemas orgánicos resaltan los del sistema nervioso, con afecciones, como encefalitis, meningitis, cauda equina e intoxicación con Diamidina (Tabla 2).

Tratamiento quirúrgico: 161 casos requirieron cirugía, principalmente, del sistema reproductivo (91,7\%), seguido por el SME $(76,4 \%)$ y SD $(64,8 \%$ ) (Figura 3); la mayoría de intervenciones quirúrgicas estuvieron relacionadas con las extremidades y el intestino grueso (Figura 4).

Figura 3. Manejo quirúrgico y mortalidad por sistema. SME: sistema músculo esquelético; SD: sistema digestivo; SRepro: sistema reproductivo; SResp: sistema respiratorio. 


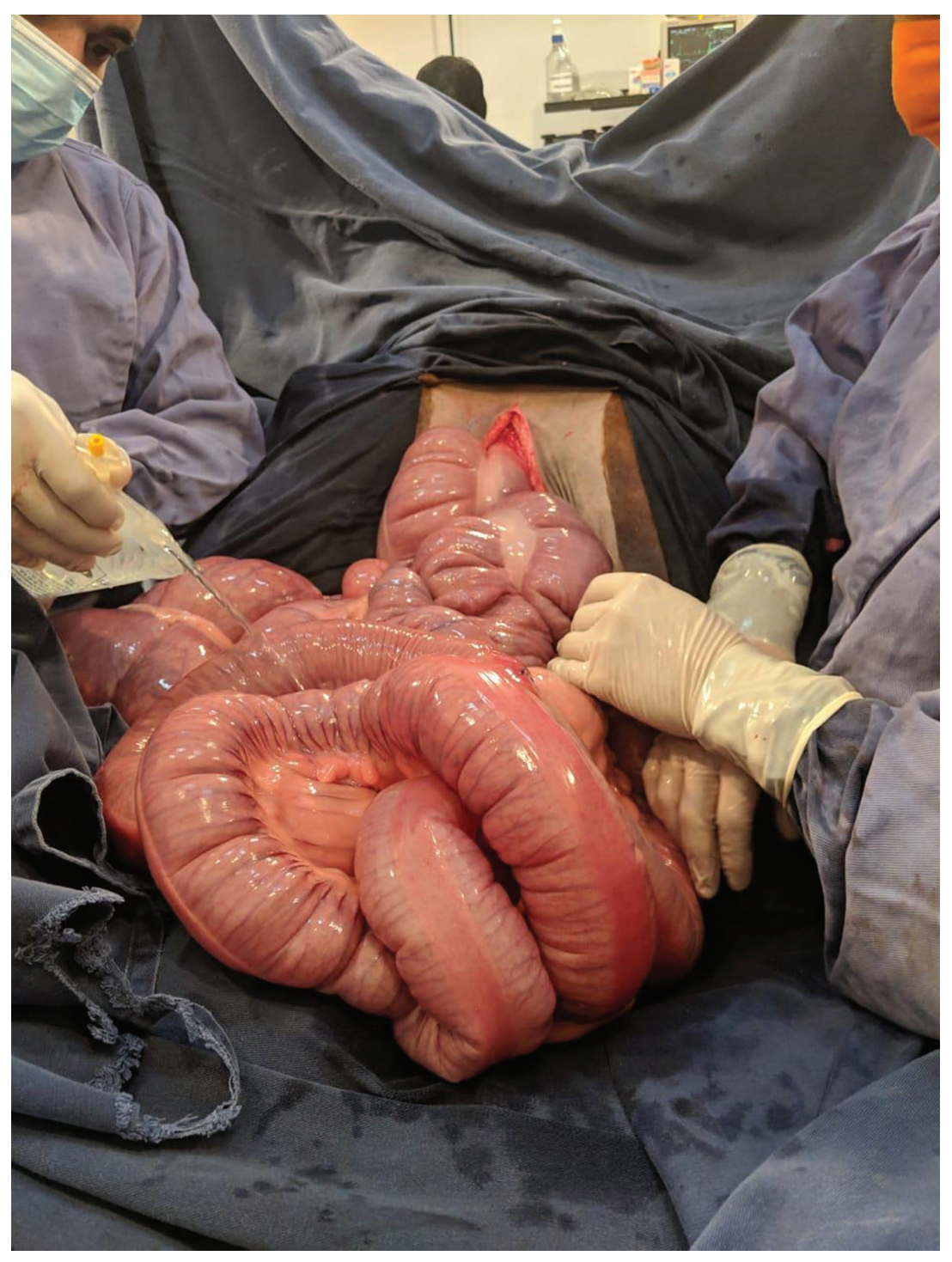

Figura 4. Enterotomía de colon menor por fecalito.

Esta casuística quirúrgica, que es similar a la de la clínica veterinaria Lasallista (Jaramillo et al. 2017), contrasta con la del ejercicio clínico ambulatorio de la Universidad de Córdoba, en la que el tratamiento quirúrgico fue bajo (5\%), siendo la piel y anexos los más intervenidos (Cardona et al. 2017). La diferencia entre el tratamiento dado a los pacientes de los centros especializados y de los servicios ambulatorios, pude deberse a la frecuencia de presentación de los padecimientos en ambos grupos de animales, influenciada por el manejo y las actividades a las que son sometidos y por el costo que implica la remisión de los casos a los centros especializados.

Mortalidad: La mortalidad total fue 13,7\%, 13 equinos fallecieron y a 22 se les practicó la eutanasia humanitaria. Por sistema, la mortalidad fue mayor en los casos del digestivo, de los cuales el 78\% recibió eutanasia, como método terapéutico, particularmente en los casos de cólico que presentan dificultad para llegar a un diagnóstico preciso y rápido (Duque et al. 2010), dado su carácter dinámico y multifactorial (Zuluaga et al. 2017).

Aunque la mortalidad del sistema ME fue baja (2,8\%), de los 3 casos fatales por eutanasia terapéutica, 2 se relacionaron con fracturas en los miembros y evidencian el nivel de compromiso que puede sufrir del aparato locomotor con estas lesiones, que son una de las principales causas de retiro temporal o total de las actividades de los caballos e, incluso, de eutanasia (Morales et al. 2009). Menor mortalidad se presentó en los sistemas reproductivo, con 2 casos $(8,3 \%)$, donde uno fue por eutanasia; nervioso e inmune, con dos fallecimientos, cada uno y, cardiovascular, urinario y multisistémico, con un caso fatal, cada uno. No se presentó mortalidad en casos del sistema respiratorio (Figura 3). 
Del presente estudio, se concluye que la casuística clínica es amplia y variada, siendo los casos más frecuentes los relacionados con los sistemas digestivo, músculo esquelético y reproductivo, por alteraciones del intestino grueso, la hiperflexión de los miembros posteriores y la criptorquia, las cuales derivaron en tratamiento quirúrgico, presentándose estas alteraciones, principalmente, en animales adultos y en hembras, a excepción de las patologías del sistema reproductivo, que afectaron más a machos y, en caballos Criollo Colombiano, probablemente, por la amplia distribución de esta raza en las fincas y en los criaderos, ubicados cerca de la Clínica U.D.C.A. La mortalidad, en un tercio de los casos relacionados con el sistema digestivo por la aplicación de eutanasia humanitaria, indica la importancia de estas patologías y la necesidad de concientizar sobre el valor de brindar atención inicial rápida y acertada, en cualquier escenario clínico, para facilitar la toma de medidas que reduzcan el sufrimiento y riesgo vital de los animales.

El presente estudio muestra los problemas clínicos a los cuales se puede ver enfrentado un médico veterinario en el ejercicio de la clínica equina y la necesidad de investigar en cuanto al Arpeo idiopático en el Caballo Criollo Colombiano y los problemas del sistema digestivo, la causa y los factores predisponentes.

Los resultados obtenidos resaltan el considerable papel de los servicios clínicos médico-quirúrgicos en equinos y motiva continuar con el fortalecimiento de estos servicios, en beneficio de la comunidad, la academia y el sector pecuario nacional.

Agradecimientos: A los Doctores Jorge Torres Vanegas y Eduardo Rubiano Rivadeneira, profesores y Médicos cirujanos de la Clínica Veterinaria U.D.C.A, por la asesoría en cuestiones médicas y de la producción equina. Al Ingeniero Alfonso Romero Paredes, profesor Ingeniería Geográfica y Ambiental U.D.C.A, por la asesoría y elaboración de la distribución geográfica de casos. A la Universidad de Ciencias Aplicadas y Ambientales U.D.C.A y su Clínica Veterinaria. Conflicto de intereses: El presente trabajo fue preparado por la autora, quien declara que no existe conflicto de intereses que ponga en riesgo la validez de los resultados o la voluntad de publicación de los mismos.

\section{REFERENCIAS}

1. AMAYA, J.; VERA, L.; SÁNCHEZ, J. 2009. Enfermedades orales más frecuentes del caballo criollo colombiano. Rev. CES Med. Vet. Zootec. 4(1):49-66.

2. ARIAS, M.P.; ZULUAGA, D.A.; BERRÍO, C.; GIRALDO, R.A.; ESCOBAR, M. 2013. Hallazgos endoscópicos de las vías respiratorias altas en equinos de la policía metropolitana de Medellín, Colombia. Rev. Med. Vet. Zoot. 60:23-31.

3. BUITRAGO-MEJÍA, J.; DÍAZ-CUETO, M.; SUÁREZCHICA, A.; CARDONA-ALVAREZ, J. 2017. Distribución geográfica de la casuística clínica equina del servicio ambulatorio de grandes animales de la Universidad de
Córdoba, Colombia. Rev. Científica, FCV-LUZ. 27(5):270281.

4. CARDONA, J.; VERGARA, J.; MARTÍNEZ, M. 2018. Prevalencia de patologías orales en caballos (Equus ferus caballus) de vaquería en el departamento de Córdoba, Colombia. REDVET. 19(5).

5. CARDONA, J.; MARTÍNEZ, M.; MAZA, L. 2017. Casuística clínica más frecuente en el servicio ambulatorio de grandes animales de la Universidad de Córdoba, Colombia. Rev. Col. Cienc. Anim. 9(1):66-72. https://doi.org/10.24188/ recia.v9.n1.2017.500

6. CASTILLO, C.; MIRA, J.; SUÁREZ, A. 2013. Presencia de obstrucción recurrente de las vías aéreas en un grupo de caballos criollos colombianos con signología respiratoria. Rev. Med. Vet. 26:37-45.

7. CRUZ, J.; SÁNCHEZ, J.; VERA, L. 2012. Caracterización y prevalencia de las enfermedades orales en el caballo criollo, departamento de Caldas, Colombia. Rev. Med. Vet. 23:3950 .

8. DUQUE, D.; SARMIENTO, L.; ARIAS, M.; CORONADO, L.; CALLE, V. 2017. Arpeo idiopático en caballos criollos colombianos: Caracterización clínica y evaluación terapéutica. Rev. Col. Cienc. Pecu. 30(supl.):59-59.

9. DUQUE, D.; VELASQUEZ, V.; ESPINOSA, L.; ARIAS, M. 2014. Idiopathic stringhalt in a Colombian Creole horse. Rev. Col. Cienc. Pec. 27(3):227-233.

10. DUQUE, D.; OSPINA, C.; ARANGO, S. 2010. Corrección quirúrgica de un atrapamiento del intestino delgado en el foramen epiploico en un caballo Pura Sangre Inglés (PSI). Rev. CES Vet. Zootec. 5(2):86-94.

11. FEDERACIÓN ECUESTRE DE COLOMBIA-FEC. 2017. Acerca de la F.E.C. Disponible desde Internet en: http:/ / www.fedecuestre.com/nosotros/acerca-de-la-f-e-c (con acceso 6/01/2019).

12. FERNANDES, C.; GUIMARÃES, C.; OLIVEIRA, B.; OLIVEIRA, G.; OLIVEIRA, C.; MEIRELLES, M.; CELEGHINI, E. 2014. Peripartum urogenital disorders in mares. Vet. Zootec. 21(4):516-526.

13. FRANCO, L.; SILVA-MOLANO, R. 2009. Frecuencia de presentación de la casuística atendida en el área de clínica quirúrgica del Hospital Veterinario de la Universidad de Caldas (2002-2004). Vet .Zootec. 3(2):51-55.

14. FREEMAN, D.; SCHAEFFER, D. 2001. Age distributions of horses with strangulation of the small intestine by a lipoma 
or in the epiploic foramen: 46 cases (1994-2000). J. Am. Vet. Med. Assoc. 219(1):87-89. https://doi.org/10.2460/ javma.2001.219.87

15. GÓMEZ T., A.; ARIZA B., F.; JIMÉNEZ, R.; ROBAYO, L. 2007. Caracterización genética de seis microsatélites en equinos colombianos Equus caballus (Equidae) y su uso en pruebas de filiación. Rev. U.D.C.A. Act. \& Div. Cient. 10(1):119-128. https://doi.org/10.31910/rudca.v10. n1.2007.574

16. HENAO, S.; TOJANCI, C.; YEPES, C.; USUGA, A. 2010. Análisis retrospectivo de los registros clínicos del Centro de Veterinaria y Zootecnia de la Universidad CES 2004-2009. Rev. CES. Med. Vet. 5(2):61-68.

17. INSTITUTO COLOMBIANO AGROPECUARIO-ICA. 2017. Censo Pecuario Nacional-2017. Disponible desde Internet en: https://www.ica.gov.co/getattachment/ Areas/Pecuaria/Servicios/Epidemiologia-Veterinaria/ Censos-2016/Censo-2017/CENSO-EQUINO-2017-1. xlsx.aspx?lang $=$ es-CO (con acceso 28/12/2018).

18. JARAMILLO, C.; CASTILLO, C.; FONSECA, B.; AGUDELO, F.; FERNÁNDEZ, J. 2017. Caracterización de las patologías presentadas en los caballos atendidos en la Clínica Veterinaria Lasallista entre 2010 y 2017. Rev. Col. Cienc. Pec. 30 (supl.):59-60.

19. LARANJEIRA P.; ALMEIDA, F.; PEREIRA, M.; LOPES, M.; CAMPOS, C.; CAIUBY, L.; SOUZA, P. 2009. Perfil e distribuição da síndrome cólica em equinos em três unidades militares do Estado do Rio de Janeiro, Brasil. Ciência Rural. 39(4):1108-1115.

20. CATTELAN, J.; MACORIS, D.; BARNABÉ, P.; URBINATI, E.; MALHEIROS, E. 2004. Criptorquismo em eqüinos: aspectos clínico-cirúrgicos e determinação da testosterona sérica. Arq. Bras. Med. Vet. Zootec. 56(2):150-156.

21. MARTÍNEZ, D.A.; OLIVER, O.J. 2012. Estudio retrospectivo de las afecciones obstructivas de las vías aéreas superiores en equinos adultos presentados a la clínica para grandes animales de la Universidad Nacional de Colombia entre 1993 y 2007. Rev. Med. Vet. Zoot. 59(3):143-150.

22. MARTÍNEZ, J.; RESENDE, F.; SILVEIRA, A. 2007. Considerações sobre a claudicação mecânica caracterizada por hiperflexão do tarso -Arpejamento-. Braz. J. Equin. Med. 2(14):22-24.
23. MENARIM, B.; VASCONCELOS, V.; CISNEROS, L.; CARNEIRO, R.; BUSCH, L.; VULCANO, L. 2012. Radiographic abnormalities in barrel racing horses with lameness referable to the metacarpophalangeal joint. J. Equine Vet. Sci. 32(4):216-221. http//:doi.org/10.1016/ j.jevs.2011.09.064

24. MINISTERIO DE SALUD. 1993. Resolución 8430 de 1993. Disponible desde Internet en: https://www.minsalud. gov.co/sites/rid/Lists/BibliotecaDigital/RIDE/DE/ DIJ/RESOLUCION-8430-DE-1993.PDF (con acceso 28/03/2019).

25. MONTOYA, S.; BOTERO, L. 2008. Caracterización ultrasonográfica de los ligamentos colaterales de la articulación metacarpo-falángica en el caballo criollo colombiano del valle de Aburrá. Rev. CES Med. Vet. Zootec. 3(2):43-50.

26. MORALES B., A.; BERMÚDEZ G., V.; GARCÍA G., F.; PERDIGÓN M., M.; NARANJO B., R. 2009. Lesiones músculo esqueléticas catastróficas en caballos pura sangre de carrera, Caracas-Venezuela. Rev. Inv. Vet. Perú. 20(2):243-248.

27. ORTIZ, O.; MARTínEZ, D.; CORREA, A.; CORREA, I. 2013. Sistematización y análisis retrospectivo de la casuística equina presentada en los años 2008 y 2009 en la Clínica Veterinaria San Luís del departamento de Antioquia, Colombia. REDVET. 14(11).

28. VEGA, F.E.; MARTÍNEZ, J.R. 2018. Diagnóstico ecográfico de las alteraciones del músculo extensor digital lateral, tendón y vaina sinovial en caballos criollos colombianos con signos clínicos de hiperflexión del tarso. Rev Col. Cienc. Pec. 31(3):188-195. https://doi.org/10.17533/udea. rccp.v31n $3 \mathrm{a} 03$

29. ZULUAGA, A.M.; SILVEIRA, G.E.; MARTÍNEZ, J.R. 2017. Consideraciones para la toma de decisiones oportunas ante el cólico equino: ¿manejo médico o quirúrgico? Rev. Med. Vet. 33:125-136. https://dx.doi.org/10.19052/mv.4060 